\title{
Helicobacter pylori-infected MSCs acquire a pro-inflammatory phenotype and induce human gastric cancer migration by promoting EMT in gastric cancer cells
}

\author{
QIANG ZHANG ${ }^{1}$, JUAN DING ${ }^{1,2}$, JINJUN LIU ${ }^{1}$, WEI WANG ${ }^{1}$, FENG ZHANG ${ }^{1}$, JUNHE WANG ${ }^{2}$ and YUYUN LI ${ }^{2}$ \\ ${ }^{1}$ Department of Clinical Laboratory Science, The First Affiliated Hospital of Bengbu Medical College, Bengbu, Anhui 233004; \\ ${ }^{2}$ Department of Clinical Laboratory, Bengbu Medical College, Bengbu, Anhui 233030, P.R. China
}

Received December 30, 2014; Accepted August 5, 2015

DOI: $10.3892 / 01.2015 .3897$

\begin{abstract}
Accumulating clinical and experimental evidence has suggested that Helicobacter pylori (H. pylori) infection-associated gastric cancer (GC) is associated with high rates of mortality and serious health effects. The majority of patients succumb to $H$. pylori infection-associated GC due to metastasis. Mesenchymal stem cells (MSCs), which have multipotent differentiation potential, may be recruited into the tumor-associated stroma. MSCs are crucial components of the H. pylori infection-associated GC microenvironment, and may be critical for GC cell migration. In this study, an MSCs/H. pylori co-culture model was designed, and the effect of $H$. pylori-infected MSCs on the migration of GC cells was evaluated using a Transwell migration assay. $H$. pylori-infected MSC cytokine expression was evaluated using Luminex/ELISA. The expression of epithelial-mesenchymal transition (EMT) markers in the GC cells treated with supernatants from $H$. pylori-infected MSCs were detected by western blot analysis. The results demonstrated that the interaction between MSCs and H. pylori may induce $\mathrm{GC}$ cell migration, through secretion of a combination of cytokines that promote EMT in GC cells. The expression of phosphorylated forms of nuclear factor- $\mathrm{\kappa B}(\mathrm{NF}-\mathrm{\kappa} \mathrm{B})$ was observed to be increased in MSCs by H. pylori. Inhibition of NF- $\mathrm{KB}$ activation by pyrrolidine dithiocarbamate blocked the effects of $H$. pylori-infected MSCs on SGC-7901 human stomach adenocarcinoma cell migration. Overall, the results
\end{abstract}

Correspondence to: Dr Qiang Zhang, Department of Clinical Laboratory Science, The First Affiliated Hospital of Bengbu Medical College, 287 Chang Huai Road, Bengbu, Anhui 233004, P.R. China

E-mail: bbyxyzq@163.com

Mrs. Yuyun Li, Department of Clinical Laboratory, Bengbu Medical College, 2600 Dong Hai Street, Bengbu, Anhui 233030, P.R. China E-mail: bbmcliyuyun@163.com

Key words: Helicobacter pylori, gastric cancer, mesenchymal stem cells, epithelial-mesenchymal transition, migration of the present study suggest that $\mathrm{H}$. pylori-infected MSCs acquire a pro-inflammatory phenotype through secretion of a combination of multiple cytokines, a number of which are NF-кB-dependent. These cytokines enhance $H$. pylori infection-associated GC cell migration by promoting EMT in GC cells. The results of the present study provide novel evidence for the modulatory effect of MSCs in the tumor microenvironment and provide insight into the significance of stromal cell involvement in GC progression.

\section{Introduction}

Helicobacter pylori (H. pylori) infection-associated gastric cancer (GC) is a common digestive tract malignancy that is associated with high rates of mortality and serious health effects $(1,2)$. Metastasis is a significant stage in GC development, and the majority of patients succumb to H. pylori infection-associated GC due to metastasis. $(2,3)$. Consequently, investigation into the mechanisms underlying GC metastasis has become a key area of GC research. Invasion and metastasis of GC tumors are thought to be the most lethal and prominent features associated with disease recurrence (4). However, the mechanisms underlying the involvement of $H$. pylori in the invasion, metastasis and recurrence of $H$. pylori infection-associated GC remain to be elucidated. Previous studies have suggested that the epithelial-mesenchymal transition (EMT) is critical for the invasion and metastasis of malignant tumors (5). EMT is associated with normal tissue development, organogenesis, tissue remodeling and wound healing (6). By contrast, aberrant EMT reactivation contributes to the initiation of numerous human pathologies, particularly those associated with certain types of solid tumor invasion and metastasis (4), including that exhibited by GC cells (7). Gaining an understanding of these mechanisms may aid the therapeutic control of EMT, in order to promote tissue regeneration, treat fibrosis and prevent cancer invasion and metastasis.

Mesenchymal stem cells (MSCs) are multipotent adult stem cells, which have been observed in multiple types of tissue $(8,9)$. MSCs have been reported to exhibit tropism for inflammation and cancer sites (10-14). In addition, $H$. pylori-induced epithelial responses may contribute to 
directing the homing of MSCs into the gastric mucosa $(15,16)$. As a major component of the $H$.pylori infection-associated GC microenvironment, MSCs may be critical for malignant tumor invasion and metastasis; however, the role of $H$.pylori-infected MSCs remains to be elucidated and is subject to controversy. In the present study, a human umbilical cord MSC (hucMSC)/H. pylori co-culture model was developed. The effects of $H$. pylori-infected hucMSCs on SGC-7901 GC cell migration were evaluated in vitro using a Transwell migration assay. During infection, MSC cytokine expression was evaluated using Luminex/ELISA, and the abilities of certain identified cytokines to induce GC cell migration were individually evaluated in vitro. Finally, the significance of the nuclear factor- $\kappa \mathrm{B}(\mathrm{NF}-\kappa \mathrm{B})$ signaling pathway in cytokine secretion was evaluated.

The results of the present study may enhance understanding of the significance of MSCs in $H$. pylori infection-associated GC and offer therapeutic benefits by inhibiting malignant processes involved in the promotion of cancer.

\section{Materials and methods}

Cell culture and H. pylori strain growth conditions. The SCG-7901 human gastric cancer cell line was purchased from the Institute of Biochemistry and Cell Biology at the Chinese Academy of Sciences (Shanghai, China). Fresh umbilical cords were collected from healthy puerperal mothers after written informed consent was obtained, and MSCs were isolated from these human umbilical cord tissues and characterized as described by Qiao et al (17). Pregnant women with pre-eclampsia, sexually transmitted diseases or hepatitis were excluded from the study. HucMSCs at passage 3 were selected for use in the present study. SGC-7901 cells and hucMSCs were cultured in Invitrogen low-glucose Dulbecco's modified Eagle's medium (L-DMEM; Thermo Fisher Scientific, Inc., Carlsbad, CA, USA) with $10 \%$ fetal bovine serum (FBS; Invitrogen; Thermo Fisher Scientific, Inc.). All cells were incubated at $37^{\circ} \mathrm{C}$ in a humidified cell culture incubator in an atmosphere of $5 \% \mathrm{CO}_{2}$. The 11673 $H$. pylori strain was provided by Dr Weng-Rong Xu (Jiangsu University, Zhenjiang, China). The H.pylori strain was grown in trypticase soy agar (QingDao Hope Bio-technology Co., Ltd., Qingdao, China) supplemented with 5\% sheep blood (QingDao Hope Bio-technology Co., Ltd.) and incubated at $37^{\circ} \mathrm{C}$ under microaerobic conditions. For the co-culture experiments, the H. pylori strain was grown for $48 \mathrm{~h}$, resuspended in L-DMEM with $10 \%$ FBS and adjusted to optical density $600 \mathrm{~nm}=1$ [corresponding to $1 \times 10^{8}$ colony-forming units $(\mathrm{CFU}) / \mathrm{ml}]$ prior to infection. All experimental protocols were approved by the Ethics Committee of Bengbu Medical College, Bengbu, China.

Co-culture of hucMSCs with H. pylori. A hucMSCs/H. pylori co-culture model was designed as previously described (18). Briefly, hucMSC cells were trypsinized (Trypsin; Amresco LLC, Solon, OH, USA), resuspended in L-DMEM with $10 \%$ FBS and seeded into a culture flask. Colonies of H. pylori $(48 \mathrm{~h})$ were collected and bacterial cells were added to the monolayer at a multiplicity of infection (MOI) of 100 bacteria/cell. Cultures were maintained in a $5 \% \mathrm{CO}_{2}$ humidified atmosphere at $37^{\circ} \mathrm{C}$ for $24 \mathrm{~h}$. The supernatants were collected and centrifuged at $800 \mathrm{x}$ g for $10 \mathrm{~min}$ at $4^{\circ} \mathrm{C}$, and were subsequently filtered through a $0.22-\mu \mathrm{m}$ membrane (EMD Millipore, Billerica, MA, USA) and stored at $-80^{\circ} \mathrm{C}$ until use. Following the collection of supernatants, PBS was replaced twice in order to remove floating hucMSCs, debris and $H$. pylori. The infected hucMSCs were then harvested and subjected to the following experiments. As a control, uninfected hucMSCs were processed in a similar manner, but in the absence of bacteria.

Transwell migration assay. The migration assay was adapted from a previously described protocol (19). Briefly, migration was measured in cell culture inserts with $8.0-\mu \mathrm{m}$ pore filters (Corning Inc., Corning, NY, USA). SGC-7901 cells $\left(5 \times 10^{4}\right.$ cells $\left./ 200 \mu \mathrm{l}\right)$ suspended in serum-free medium (Invitrogen; Thermo Fisher Scientific, Inc.) were loaded into the upper compartment of a Transwell chamber and $600 \mu \mathrm{l}$ $10 \%$ FBS-L-DMEM containing hucMSCs $\left(5 \times 10^{4}\right.$ cells/well $)$ in the presence or absence of H.pylori (MOI, 100:1) was added to the lower well of the Transwell chamber (Fig. 1A). Following incubation for $8 \mathrm{~h}$, SGC-7901 cells which remained at the bottom of the polycarbonate membrane were removed using cotton swabs. Cells which migrated to the lower surface of the membrane were fixed with $4 \%$ paraformaldehyde (AR-0211; DingGuo Biotechnology Co., Ltd, Shanghai, China) for $30 \mathrm{~min}$. The migrated cells were subsequently stained with crystal violet (C3886; Sigma-Aldrich, Shanghai, China) for $15 \mathrm{~min}$ and counted in 10 random fields under a microscope (TE300; Nikon Corporation, Tokyo, Japan). Each assay was repeated three times.

Luminex assay/ELISA. The concentrations of interleukin (IL)-8, IL-6, granulocyte macrophage colony-stimulating factor (GM-CSF), platelet-derived growth factor-B (PDGF-B), monocyte chemoattractant protein-1 (MCP-1), vascular endothelial growth factor (VEGF), epidermal growth factor (EGF), tumor necrosis factor- $\alpha$ (TNF- $\alpha$ ), IL-10, IL-1 $\beta$, IL-15, IL-2 and IL-17 in the supernatants of $H$. pylori-infected hucMSCs were evaluated by Luminex assay (Cytokine and Chemokine Magnetic Bead Panel kit; \#HCYTOMAG -60K; Merck Millipore, Darmstadt, Germany) or human IL-6 ELISA Kit (DKW12-1060-096), human IL-8 ELISA Kit (DKW12-1080-096) or human PDGF-BB ELISA Kit (GWB-SKR056; all Dakewe Biotech Co., Ltd., Shenzhen, China), according to the manufacturer's instructions. The Luminex assay detected the expression of cytokines in the supernatants from hucMSCs and $H$. pylori-infected hucMSCs. All procedures were processed according to the manufacturer's instructions. The ELISA assay was subsequently used to evaluate IL-6, IL-8 and PDGF-B levels, as these were observed to be the most highly expressed cytokines in the supernatants of hucMSCs and $H$. pylori-infected hucMSCs. The signals were detected and analyzed by using the Luminex 200 system (Merck Millipore).

Western blot analysis. Western blot analysis was adapted as previously described (19). Cells were lysed in radioimmunoprecipitation assay buffer (Beyotime Institute of Biotechnology, Shanghai, China) supplemented with complete 
protease inhibitors (Shanghai Haoran Biotechnology Co., Ltd., Shanghai, China) on ice. Aliquots containing identical amounts of protein were fractionated by SDS-PAGE (Beyotime Institute of Biotechnology) and then transferred onto polyvinylidene difluoride membranes (EMD Millipore) following electrophoresis. After blocking in $5 \%(\mathrm{w} / \mathrm{v})$ non-fat milk for $1 \mathrm{~h}$ at room temperature, the membranes were incubated at their respective appropriate dilutions of specific primary antibodies overnight at $4^{\circ} \mathrm{C}$. Following incubation with the secondary antibodies for $2 \mathrm{~h}$ at $37^{\circ} \mathrm{C}$, the signal was visualized using horseradish peroxidase (HRP) substrate (EMD Millipore) and analyzed using MD ImageQuant ${ }^{\mathrm{TM}}$ Software (G:Box; Syngene, Cambridge, UK). The following rabbit anti-human primary antibodies were used for the western blot analysis: Anti-IкB- $\alpha$ (polyclonal IgG; dilution, 1:500; cat. no. sc-371; Santa Cruz Biotechnology, Inc., Dallas, TX, USA); anti-p-NF-кB-p65 (polyclonal IgG; dilution, 1:500; cat. no. sc-101749; Santa Cruz Biotechnology, Inc.); anti-t-NF- $\mathrm{B}-\mathrm{p} 65$ (monoclonal IgG; dilution, 1:1,000; cat. no. sc-372; Santa Cruz Biotechnology, Inc.); anti-N-cadherin (polyclonal IgG; dilution, 1:800; cat. no. BS2224; Bioworld Technology, Inc., St. Louis Park, MN, USA); anti-E-cadherin (polyclonal IgG; dilution, 1:1,000; cat. no. BS1097; Bioworld Technology, Inc.); anti-Vimentin (polyclonal IgG; dilution, 1:500; cat. no. BS1855; Bioworld Technology, Inc.); anti- $\beta$-actin (polyclonal IgG; dilution, 1:10,000; cat. no. AP0060; Bioworld Technology, Inc.); and anti-histone (polyclonal IgG; dilution, 1:1,000; cat. no. BS1160; Bioworld Technology, Inc.). HRP-conjugated goat anti-rabbit polyclonal IgG secondary antibodies (dilution, 1:2,000; cat. no. L3012-2; Signalway Antibody Co., Ltd., Nangjing, China) were used.

Wound healing assay. SGC-7901 cells were allowed to grow to $50 \%$ confluence and subsequently wounded by making a single scratch in the monolayer using a pipette tip (0.1-10 $\mu \mathrm{l}$; NICHIRYO Co., Ltd., Koshigaya, Japan). Following scratching, the medium was replaced in order to remove floating cells and debris, and cells were incubated at $37^{\circ} \mathrm{C}$ for $48 \mathrm{~h}$ to allow for growth and closure of the wound. Inverted microscopic observations (magnification, x100; TE300; Nikon Corporation) were used to assess cell migration into the scratched area. Images were captured at identical positions relative to the wound at 0 and $48 \mathrm{~h}$.

GC cell incubation with cytokines. To determine whether increased cytokine expression in $H$. pylori-infected hucMSCs affected GC cell migration, specific cytokines (IL-8, IL-6 and PDGF-B; R\&D Systems, Inc., Minneapolis, MN, USA) were incubated with the SCG-7901 GC cell line in cell culture inserts. Cell migration was assessed as already described. Briefly, SGC-7901 cells $\left(5 \times 10^{4}\right.$ cells/200 $\left.\mu \mathrm{l}\right)$ were placed into the top chamber, and medium containing $10 \% \mathrm{FBS}$ and $50 \mathrm{ng} /$ ml uman cytokines (IL-6, IL-8 and PDGF) was placed into the bottom chamber of the Transwell plates $(8-\mu \mathrm{m}$ pore size; Corning Inc.). Following incubation at $37^{\circ} \mathrm{C}$ in $5 \% \mathrm{CO}_{2}$ for $8 \mathrm{~h}$, the cells remaining on the upper surface of the membrane were removed. Cells on the lower surface of the membrane were fixed and stained with crystal violet. The migration ability of the cells was determined by counting the cells under a microscope in at least 10 fields per assay.
hucMSC pre-treatment with pyrrolidine dithiocarbamate $(P D T C)$. To further investigate whether NF- $\kappa \mathrm{B}$ is involved in hucMSC pro-inflammatory phenotype differentiation in response to $H$. pylori infection and in $H$. pylori-induced hucMSC cytokines expression, hucMSCs were cultured in L-DMEM medium containing PDTC (10 $\mu \mathrm{mol} / \mathrm{ml}$; Jingmei BioTech Co., Ltd., Shanghai, China), and were then co-cultured with the $H$. pylori strain 11673 (MOI, 100:1) at $37^{\circ} \mathrm{C}$ in a humidified atmosphere of $5 \% \mathrm{CO}_{2}$ for $30,60,120$ or $180 \mathrm{~min}$, or $24 \mathrm{~h}$. SGC-7901 cells were exposed to supernatant from hucMSCs, $H$. pylori-infected hucMSCs, or $H$. pylori-infected PDTC-pre-treated hucMSCs for $48 \mathrm{~h}$ at $37^{\circ} \mathrm{C}$.

Statistical analysis. Statistical analyses were conducted using SPSS software version 16.0 (SPSS Inc., Chicago, IL, USA). Quantification values are presented as the mean \pm standard deviation of three or more independent experiments, each performed in duplicate or triplicate as indicated. Differences between groups were analyzed using one-way analysis of variance. Differences between pyrrolidine dithiocarbamate (PDTC) treatments were analyzed using a Student's t-test. $\mathrm{P}<0.05$ was considered to indicate a statistically significant difference.

\section{Results}

H. pylori-infected hucMSCs induce an EMT state in GC cells in vitro. In order to determine the effect exerted on GC cells by $H$. pylori-infected hucMSCs, morphological alterations in SGC-7901 GC cells cultured with H.pylori (11673; MOI, 100:1), or with the supernatants from hucMSCs or $H$. pylori-infected hucMSCs, were observed. Infecting hucMSCs with $H$. pylori significantly enhanced their ability to induce SGC-7901 cells to acquire a fibroblastic phenotype $(\mathrm{P}=0.0079$; data not shown). Downregulation of E-cadherin, a universal epithelial marker, is an early indicator of EMT (5). Western blot analysis of E-cadherin expression levels revealed that E-cadherin protein levels were decreased in SGC-7901 cells cultured with supernatants from $H$. pylori-infected hucMSCs, hucMSCs or those treated with $H$. pylori, compared with those cultured alone. $H$. pylori-infected hucMSCs exerted a more marked effect on E-cadherin expression than that of $H$. pylori or uninfected hucMSCs (Fig. 1B). The observed decrease in E-cadherin expression levels coincided with induction of the expression of $\mathrm{N}$-cadherin and vimentin (Fig. 1B).

Migration of SGC-7901 GC cells is enhanced in the presence of H. pylori-infected hucMSCs. Dissemination of cancer cells during tumor metastasis, a process which is enabled by EMT, requires enhancement of cell migration ability. In light of the observation that $H$. pylori-infected hucMSCs induce an EMT state in SGC-7901 cells, it was hypothesized that H. pylori-infected hucMSCs may promote SGC-7901 cell migration. To test this hypothesis, a Transwell migration assay was performed (Fig. 1A, C and D). The basal migration rate of SGC-7901 cells in the presence of the medium alone was identical to that in the presence of $H$. pylori strains alone, indicating that the presence of $H$. pylori alone was unable to stimulate SGC-7901 cell migration (Fig. 1Cb and Db). hucMSCs alone were observed to stimulate SGC-7901 cell 
A

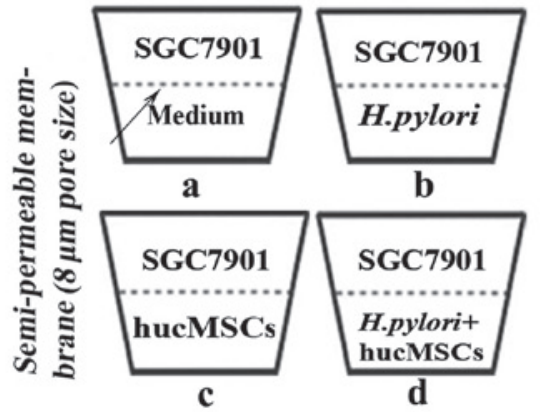

C

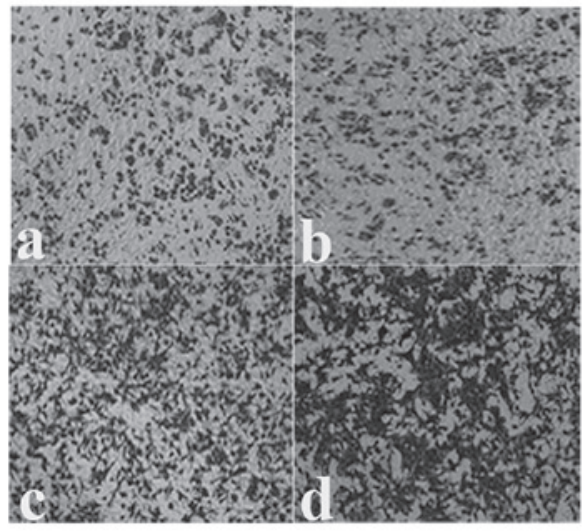

B

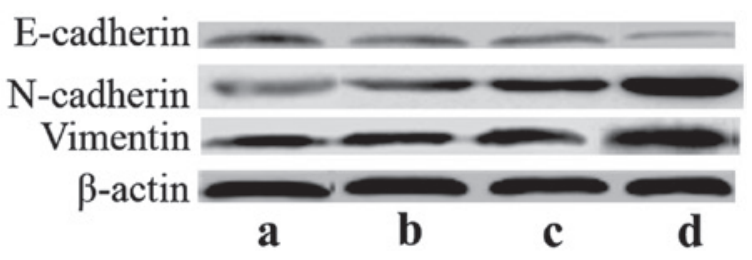

D

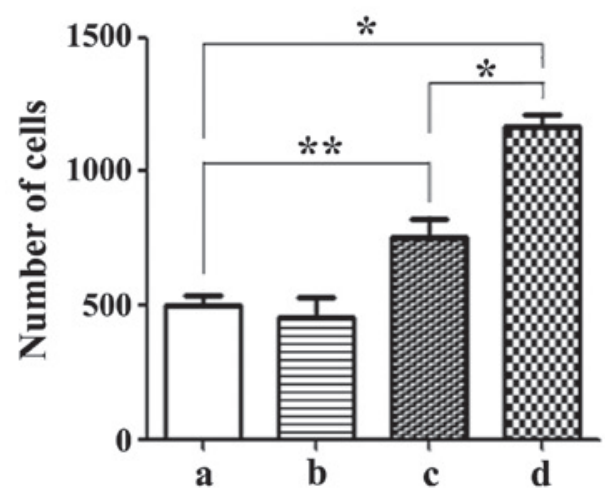

d

$\mathbf{E}$

a

b

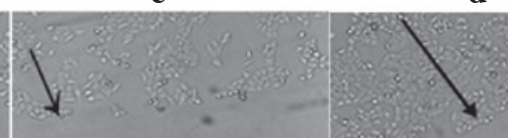

$\mathbf{0 h}$

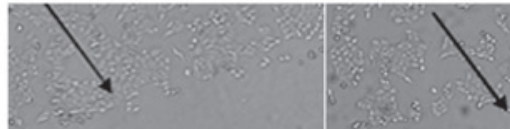

$(2)$
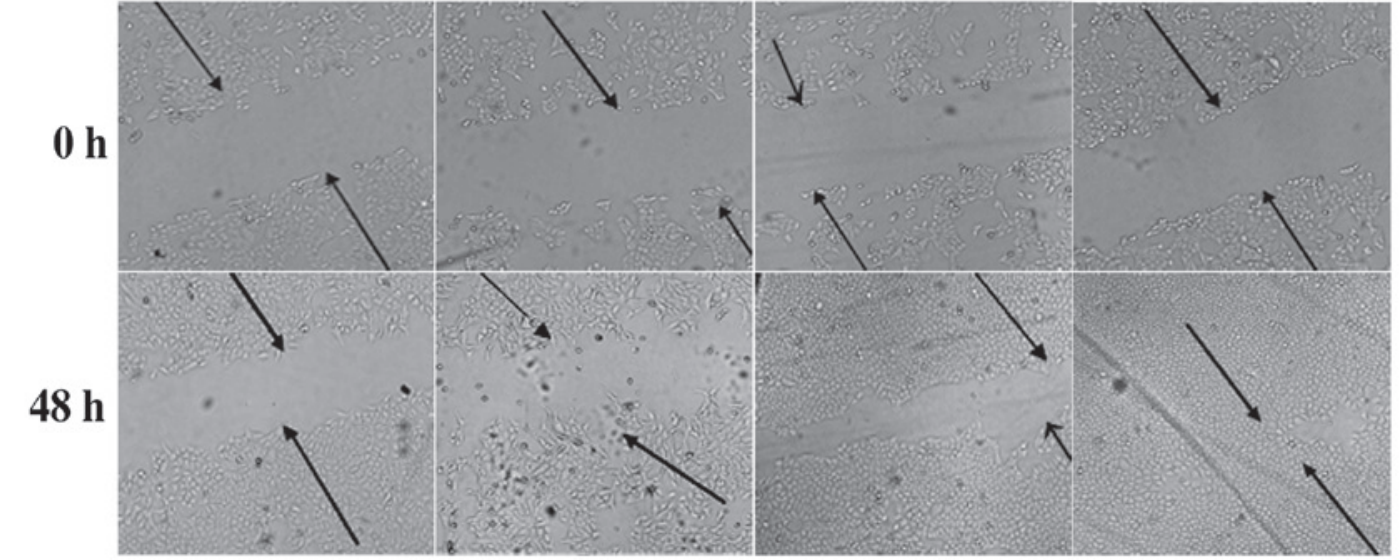

Figure 1. H. pylori-infected hucMSCs promote the migration and epithelial-mesenchymal transition of SGC-7901 cells. (A) The ability of hucMSCs to induce SCG-7901 gastric cancer cell migration following H. pylori-infection was evaluated by Transwell migration assay. (B) Western blot analysis of E-cadherin, $\mathrm{N}$-cadherin and vimentin protein levels. (C) Representative images of Transwell migration assay (magnification, x100) and (D) histogram of the numbers of migrated SGC-7901 cells in each treatment group. ${ }^{*} \mathrm{P}<0.01$ and ${ }^{* *} \mathrm{P}<0.05(\mathrm{n}=3)$. Values are presented as the mean \pm standard deviation and are representative of three independent experiments. (E) The capacity of cells to migrate to fill a scratched area devoid of cells was assessed in co-cultures. $H$. pylori-infected hucMSCs more effectively enhanced SGC-7901 cell membrane penetration and migration. (a) SCG-7901 cells alone and with (b) $H$. pylori or the supernatants from (c) hucMSCs and (d) hucMSCs infected with H. pylori. H. pylori, Helicobacter pylori; hucMSCs, human umbilical cord mesenchymal stem cells.

migration ( $\mathrm{P}=0.0255)$. However, H. pylori-infected hucMSCs markedly promoted SGC-7901 cell migration (Fig. 1Cd), compared with SGC-7901 cells alone $(\mathrm{P}=0.003)$ or with hucMSCs $(\mathrm{P}=0.0062)$ (Fig. 1Da and Dc). The number of cells that had migrated was subsequently quantified (Fig. 1D).

A wound-healing assay was performed to evaluate cell migration ability. Following scratching, cells were allowed to recover and their capacity to migrate and fill the area devoid of cells was assessed. When SGC-7901 cells were co-cultured with $H$. pylori or each type of supernatant, inverted microscopic observations revealed that the size of the scratched area decreased within $48 \mathrm{~h}$. These observations also indicated that, although SGC-7901 cells cultured under all four conditions migrated into the scratched area, only those cultured with supernatants from $H$. pylori-infected hucMSCs exhibited marked migratory behavior (Fig. 1E). H. pylori-infected hucMSCs more effectively enhanced SGC-7901 cell membrane penetration and migration.

H. pylori infection upregulates the expression of inflammatory cytokines in MSCs and a number of these promote GC cell migration. To investigate the effect of $H$. pylori on MSCs in vitro and identify the secreted cytokines in $H$.pylori-infected MSCs, hucMSC cells were infected with $H$.pylori 11673 strains as described. A Luminex assay was conducted to determine the expression levels of inflammatory-associated functional 
A

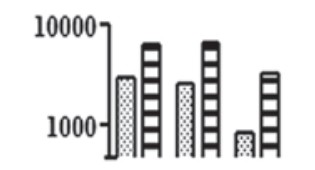

Control

$\square$ H. pylorithucMSCs (MOI=100 $24 \mathrm{~h}$ )
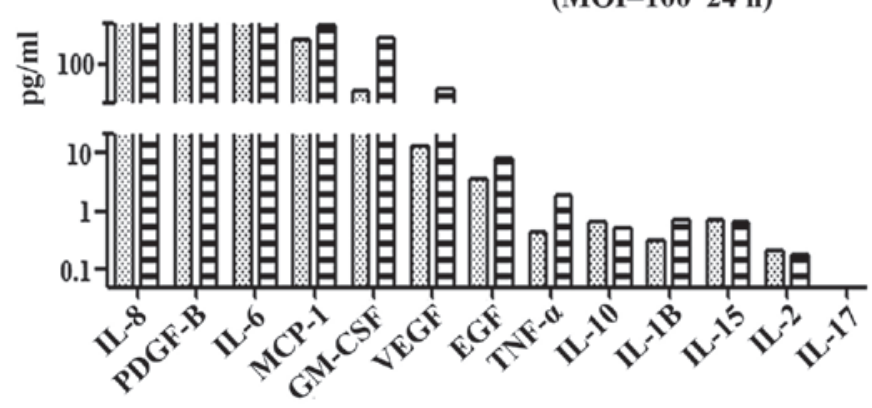

$\mathbf{B}$

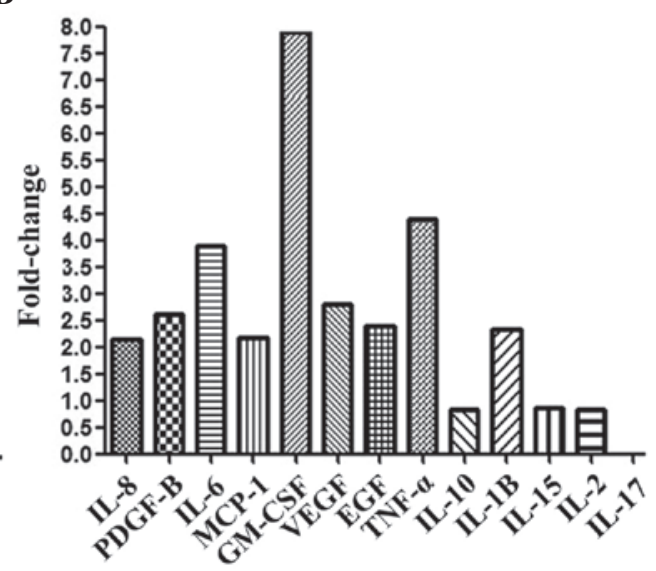

C
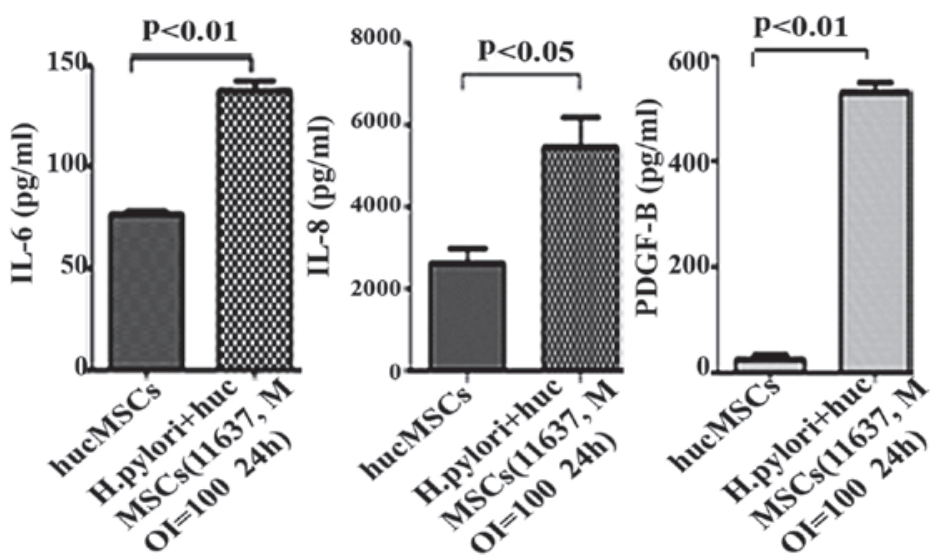

D

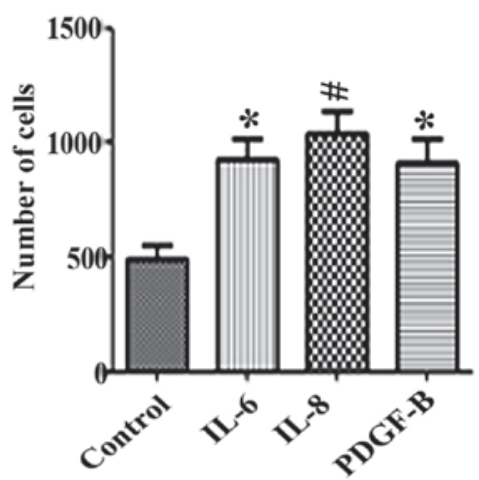

Figure 2. Expression of inflammatory cytokines in hucMSCs is upregulated following co-culture with $H$.pylori, and the cytokines produced during co-culture promote migration of SGC-7901 cells. (A) Luminex assay for the expression of cytokines in the supernatants of hucMSCs and $H$. pylori-infected hucMSCs. (B) The fold-changes in cytokine expression in H. pylori-infected humMSCs relative to uninfected cells were as follows: IL-8, 2.14; PDGF-B, 2.61; IL-6, 3.9; MCP-1, 2.17; GM-1, 8.0; VEGF, 2.8; EGF, 2.4; TNF- $\alpha$, 4.4; IL-1 $\beta, 2.35$. Fold-change = concentration of cytokine in $H$. pylori-infected humMSCs/uninfected humMSCs. (C) ELISA assay to determine IL-6, IL-8 and PDGF-B levels in the supernatants of hucMSCs and H. pylori-infected hucMSCs. (D) SGC-7901 cells were stimulated by $50 \mathrm{ng} / \mathrm{ml}$ concentrations of purified cytokines and migration assays were then performed. "P $<0.05$ and ${ }^{\#} \mathrm{P}<0.01$ compared with migration medium alone. Values are presented as the mean \pm standard deviation. Data are representative of three independent experiments. H. pylori, Helicobacter pylori; hucMSCs, human umbilical cord mesenchymal stem cells; IL, interleukin; PDGF, platelet-derived growth factor; MCP, monocyte chemoattractant protein; GM-CSF, granulocyte macrophage colony-stimulating factor; VEGF, vascular endothelial growth factor; EGF, epidermal growth factor; TNF, tumor necrosis factor; MOI, multiplicity of infection.

factors in the supernatants of $H . p y l o r i$-infected hucMSCs. The cytokines examined were selected as they are pro-inflammatory factors (20-22) and have previously been observed to be overexpressed in $H$. pylori-infected hucMSC cells. The results of the present study revealed that the expression levels of IL-8 $(\mathrm{P}=0.0095), \mathrm{PDGF}-\mathrm{B}(\mathrm{P}=0.0238), \mathrm{IL}-6(\mathrm{P}=0.0128), \mathrm{GM}-\mathrm{CSF}$ $(\mathrm{P}=0.0082), \mathrm{MCP}-1 \quad(\mathrm{P}=0.0449), \mathrm{VEGF}(\mathrm{P}=0.0472), \mathrm{EGF}$ $(\mathrm{P}=0.0360), \mathrm{IL}-1 \beta(\mathrm{P}=0.0313)$ and $\mathrm{TNF}-\alpha(\mathrm{P}=0.0367)$ were markedly increased in the supernatants of $H$. pylori-infected hucMSCs (Fig. 2A and B), while low levels of these cytokines were observed in hucMSCs that were cultured alone (Fig. 2A and B). To further confirm the increased expression of these cytokines, an ELISA was performed to examine the levels of three of these cytokines (IL-6, IL-8 and PDGF-B). It was revealed that, consistent with the results of the Luminex assay, co-incubation with $H$. pylori upregulated the expression of IL-6 $(\mathrm{P}=0.0059)$, IL-8 $(\mathrm{P}=0.023)$ and PDGF-B $(\mathrm{P}=0.0072)$ in hucMSCs (Fig. 2C). To determine whether increased cytokine expression in $H$. pylori-infected hucMSCs affected GC cell migration, specific cytokines (IL-8, IL-6 and
PDGF-B) were incubated with the SCG-7901 GC cell line in cell culture inserts for $8 \mathrm{~h}$. The results revealed that the number of migrated SGC-7901 cells was markedly increased following incubation with each of these cytokines (IL-6, P=0.0163; IL-8, $\mathrm{P}=0.0087$; PDGF-B, $\mathrm{P}=0.0244)$, compared with SCG-7901 cells alone (Fig. 2D).

H. pylori enhances the expression of certain cytokines in MSCs through the NF- $\kappa B$ pathway. In order to explore the mechanisms responsible for the promotion of cytokine secretion induced by $H$. pylori infection of MSCs, the expression of $\mathrm{NF}-\kappa \mathrm{B}$, one of several key signaling transducers involved in inflammation and cancer (23), was determined in $H$. pylori-infected MSCs. Western blotting revealed that the expression levels of NF- $\kappa \mathrm{B}-\mathrm{p} 65$ were significantly higher in hucMSCs that had been infected with H. pylori (Fig. 3A and $\mathrm{B})$ compared with that of hucMSCs cultured alone $(\mathrm{P}=0.0427)$. To further determine whether $\mathrm{NF}-\kappa \mathrm{B}$ had a major role in the function of $H$. pylori-infected hucMSCs, hucMSCs were pretreated with PDTC to in order to inhibit $\mathrm{NF}-\kappa \mathrm{B}$ 
A

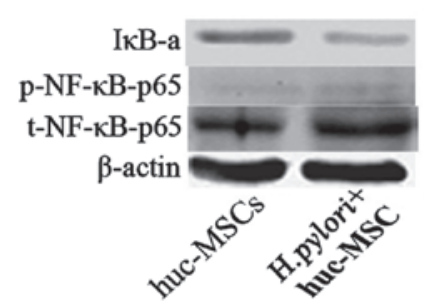

B

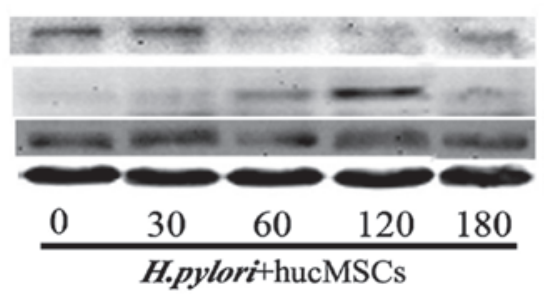

C

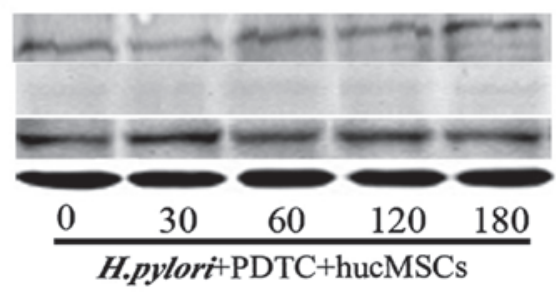

D $\mathbf{a}$

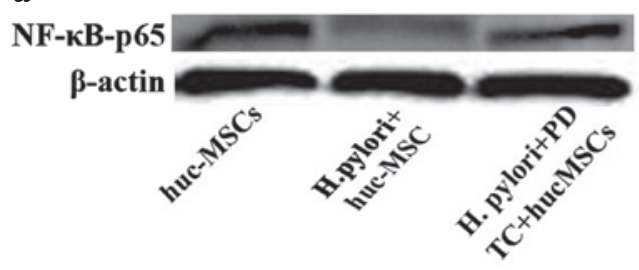

b NF-кB-p65
Histone

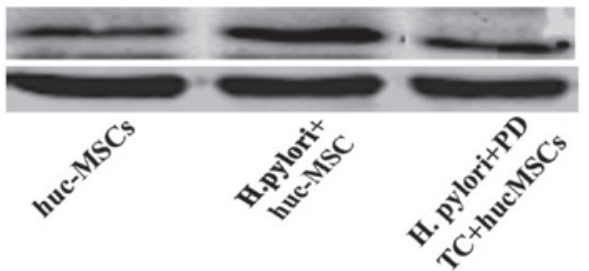

$\mathbf{E}$
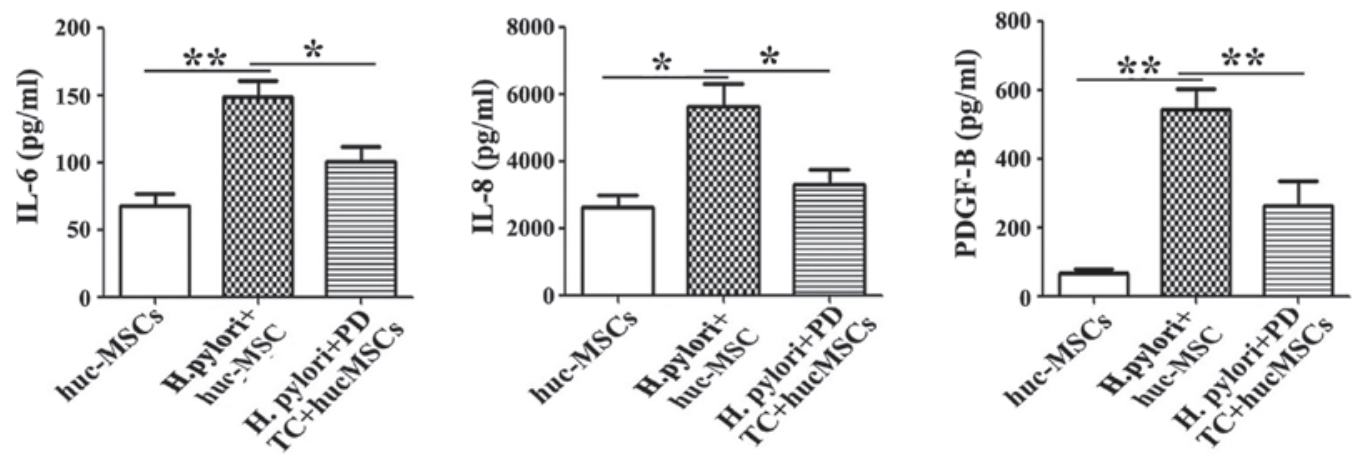

Figure 3. hucMSCs infected with $H$. pylori enhance SCG-7901 gastric cancer cell migration via NF- $\kappa$ B activation. (A) $H$. pylori-induced NF- $\kappa$ B-p65 phos-

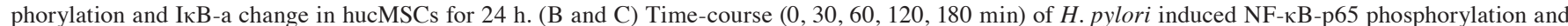
IкB-a change in hucMSCs treated with $H$. pylori in the presence or absence of PDTC (100nM). (D) Western blot analysis of (a) cytoplasmic protein and (b) nucleoprotein expression levels. (E) hucMSCs were pre-incubated with PDTC for 90 min, stimulated with $H$. pylori for 24 h and then the concentration of cytokines in the cultured medium was determined by ELISA. The NF- $\kappa$ B inhibitor PDTC was able to inhibit the expression of numerous cytokines (IL-6, IL- 8 and PDGF-B) in the hucMSCs infected with H. pylori. Data are expressed as the mean \pm standard deviation; ${ }^{*} \mathrm{P}<0.05,{ }^{* *} \mathrm{P}<0.01$; data are representative of three independent experiments. H. pylori, Helicobacter pylori; hucMSCs, human umbilical cord mesenchymal stem cells, PDTC, pyrrolidine dithiocarbamate; IL, interleukin; PDGF, platelet derived growth factor; I $\mathrm{B}-\mathrm{a}$, nuclear factor- $\kappa \mathrm{B}$ polypeptide gene enhancer; NF- $\mathrm{B}$, nuclear factor- $\kappa \mathrm{B}$; $\mathrm{p}-\mathrm{NF}-\kappa \mathrm{B}$, phosphorylated $\mathrm{NF}-\kappa \mathrm{B} ; \mathrm{t}-\mathrm{NF}-\kappa \mathrm{B}$, total NF- $\mathrm{B}$.

phosphorylation, and subsequently incubated with $H$. pylori. It was revealed that induction of $\mathrm{NF}-\kappa \mathrm{B}$ phosphorylation by H. pylori in hucMSCs was markedly inhibited by PDTC (Fig. 3C; $\mathrm{P}=0.0040$ ). The activation of the $\mathrm{NF}-\kappa \mathrm{B}$ pathway during $H$. pylori infection was confirmed by western blotting, which indicated p65 subunit translocation from the cytosol to the nucleus (Fig. 3D). To determine the role of the $\mathrm{NF}-\kappa \mathrm{B}$ pathway in the production of the cytokines responsible for SGC-7901 cell migration, IL-6, IL-8 and PDGF-B expression levels were measured in the supernatants of PDTC-pretreated $H$. pylori-infected hucMSCs. The overexpression of cytokines observed in $H$. pylori-infected hucMSCs, was abrogated in PDTC-pretreated hucMSC cells (IL-6, P=0.041; IL-8, $\mathrm{P}=0.044$; PDGF-D, $\mathrm{P}=0.031$ ), as indicated in Fig. 3E.

PDTC-pretreated MSCs reverse the migration ability of $G C$ cells. Transwell migration and wound-healing assay observations indicated that the enhanced migrations of SGC-7901 cells, facilitated by co-culture with $H$. pylori-infected MSCs, was significantly reduced in the PDTC-pretreated group (Fig. 4A and B, P=0.039; Fig. 4C, $\mathrm{P}=0.011)$. Furthermore, PDTC pretreatment also reduced the ability of $H$. pylori-infected MSC to induce EMT (defined as induction of vimentin and $\mathrm{N}$-cadherin expression and suppression of E-cadherin expression) in SCG-7901 cells in vitro (Fig. 4D).

\section{Discussion}

MSCs are multipotent adult stem cells that have been observed in multiple inflammation and cancer sites $(11,12)$. The tumor-homing properties of MSCs make them ideal candidates for use as antitumor agent delivery vehicles (24). There has also been increasing interest in understanding the role and fate of MSCs during tumor progression. Korkaya et al (25) suggested that the stromal microenvironment may be critical for regulating tumor development in the 'seed and soil' hypothesis. Among the stromal cells, MSCs communicate with cancer cells in order to induce tumor growth and enhance metastatic potential. In addition, several studies have indicated that MSCs may enhance tumor metastasis $(26,27)$, however the mechanism underlying the promotion of $H$. pylori infection-associated GC cell migration has remained to be elucidated. In the present study, hucMSCs were treated with $H$. pylori to mimic 
A

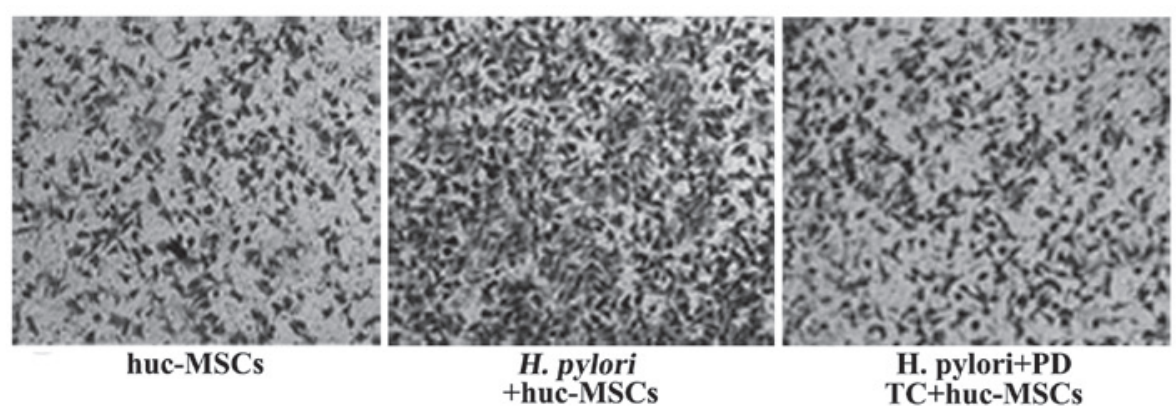

B

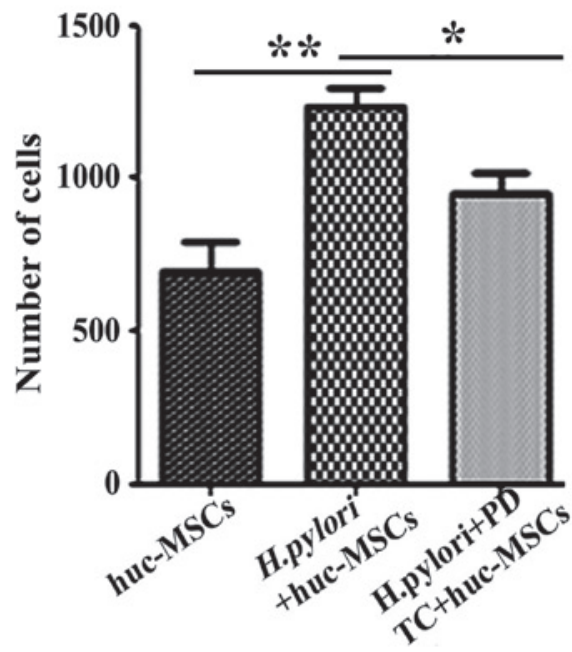

C
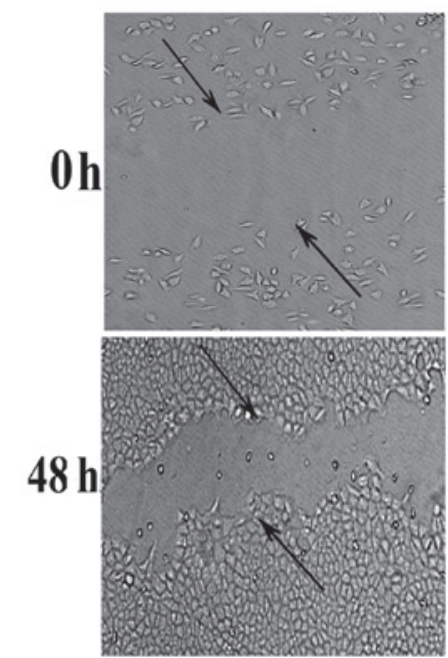

b
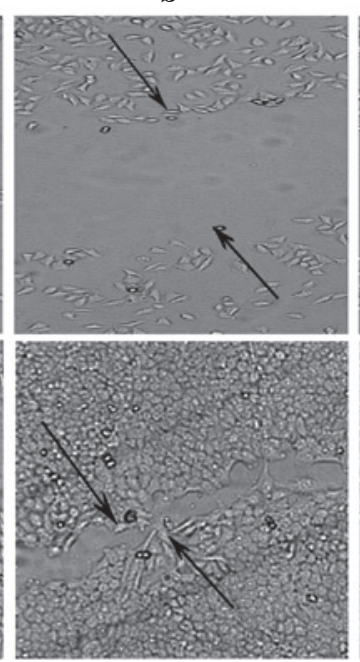

c

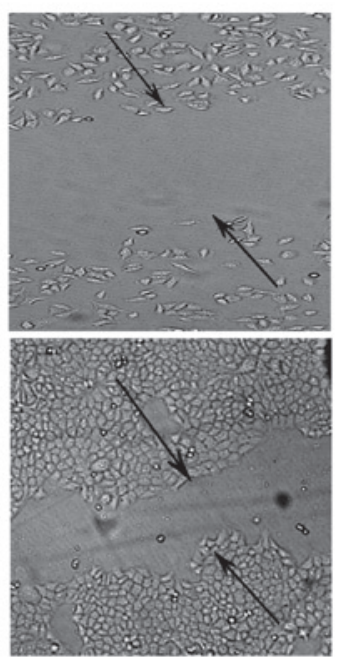

D

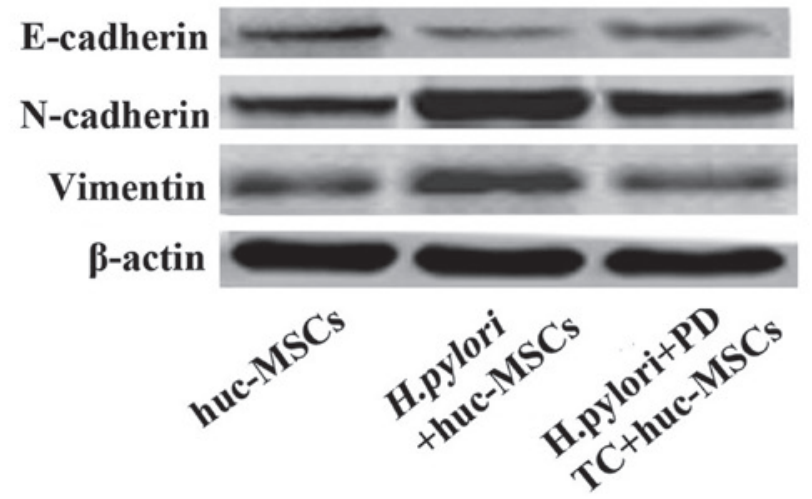

Figure 4. Inhibition of NF- $\kappa \mathrm{B}$ activation by PDTC abrogates the effect of $H$.pylori-infected MSCs on gastric cancer cells. (A) The ability of $H$. pylori-infected MSCs to induce migration following pretreatment with PDTC was evaluated using cell culture inserts; magnification, x100. (B) Histogram indicating the number of migrated SGC-7901 cells. Data are expressed as the mean \pm standard deviation; ${ }^{*} \mathrm{P}<0.05$ and ${ }^{* *} \mathrm{P}<0.01$. Data are representative of three independent experiments. (C) Capacity of cells to migrate to fill a scratched area devoid of cells was assessed in co-cultures of SCG-7901 cells with supernatants from (a) hucMSCs, (b) H. pylori-infected hucMSCs and (c) H. pylori-infected hucMSCs pretreated with PDTC for 48 h, respectively. (D) Western blot analyses of E-cadherin, N-cadherin and vimentin protein levels in SGC-7901 cells treated with the supernatants from hucMSCs, $H$. pylori-infected hucMSCs and the infected hucMSCs pretreated with PDTC, respectively. H. pylori, Helicobacter pylori; hucMSCs, human umbilical cord mesenchymal stem cells, PDTC, pyrrolidine dithiocarbamate; NF- $\kappa \mathrm{B}$, nuclear factor- $\kappa \mathrm{B}$.

the $H$. pylori infection-associated GC microenvironment and thus determine whether $H$. pylori-infected MSCs contribute to GC cell invasion and metastasis. The results of the present study suggested that short-term infection with $H$. pylori may induce MSCs to acquire a 'pro-inflammatory phenotype' and become part of the tumor microenvironment through the production of increased levels of inflammatory cytokines.

Metastasis is a common clinical finding in numerous types of human cancer, and the majority of patients with cancer succumb to the disease due to metastases (28). EMT is critical in the occurrence of tumor metastasis (4,29-31). EMT is defined as a biological process, during which epithelial cell-cell adhesion is reduced due to downregulation of adhesion molecules, including E-cadherin. Additionally, cell morphology becomes fibroblast-like due to the upregulation of vimentin and $\mathrm{N}$-cadherin (32). Studies have indicated that improper EMT has been implicated as a factor required for tumor progression through invasion and metastatic spread $(4,28)$, and that EMT 
additionally protects tumor cells against apoptosis $(32,33)$. In the present study, it was observed that $H$. pylori-infected MSCs induced EMT in GC cells and markedly increased GC cell migration. To further explain this result, the effects of certain cytokines (IL-6, IL-8 and PDGF-B), which were particularly highly expressed by infected hucMSCs, on GC cell EMT, invasion and metastasis were evaluated. The results of the present study revealed that these cytokines were able to alter the migration and EMT of GC cells. Taken together, these data suggest that $H$. pylori-infected MSCs secreted higher levels of inflammatory cytokines, which enhance GC cell invasion and metastasis abilities by promoting the occurrence of EMT.

In order to explore the mechanism responsible for the promoting role of $H$. pylori-infected MSCs in $H$. pylori infection-associated GC cell migration, the expression of the key signaling transducer for inflammation and cancer in MSCs, NF- $\kappa \mathrm{B}$, was observed (34). The results of the present study demonstrated that the levels of p-NF- $\kappa \mathrm{B}-\mathrm{p} 65$ were markedly increased in $H$. pylori-infected MSCs compared with those in the other experimental groups, and the induction of $\mathrm{NF}-\kappa \mathrm{B}$ phosphorylation by $H$. pylori in MSCs was markedly inhibited by PDTC. PDTC additionally inhibited the upregulation of IL-6, IL-8 and PDGF-B in H. pylori-infected MSCs. PDTC-pretreatment of hucMSCs reversed the ability of hucMSCs to induce GC cell EMT, invasion and metastasis when infected with $H$. pylori 11673 strains. These results further confirmed the critical role of these cytokines in SGC-7901 cell migration and their NF- $\kappa \mathrm{B}$-dependent secretion by MSCs in response to $H$. pylori infection.

In conclusion, the results of the present study demonstrated that the interaction of $H$. pylori with MSCs may effectively induce the migration of $\mathrm{GC}$ cells due to the secretion of a combination of cytokines, a number of which are $\mathrm{NF}-\kappa \mathrm{B}$ dependent. These results also indicated that $H$. pylori-infected MSCs promote GC cell migration by promoting the occurrence of EMT. The present study provides evidence that H. pylori-infected MSCs are able to activate the migratory properties of GC cells, suggesting the existence of communication between GC cells and $H$. pylori-infected MSCs in the gastric mucosa via cytokine production. The results of the present study may contribute to the development of viable strategies for the design of novel targeted therapies, in order to prevent the formation of distant metastases of $H$. pylori infection-associated GC cells.

\section{Acknowledgments}

The present study was supported by the Major Research Plan of the National Natural Science Foundation of China (grant no. 91129718), the National Natural Science Foundation of China (grant nos. 81071421, 81000181 and 81201660) and Anhui Province's Natural Science Foundation (grant no. SBK201342044).

\section{References}

1. Liu GM, Zhou C, Xie C, Yang Z and Lv NH: Recent advances in research of gastric cancer stem cells. World Chinese Journal of Digestology 7: 574-579, 2012 (In Chinese).

2. Lee YY and Derakhshan MH: Environmental and lifestyle risk factors of gastric cancer. Arch Iran Med 16: 358-365, 2013.
3. Lee KE, Khoi PN, Xia Y, Park JS, Joo YE, Kim KK, Choi SY and Jung YD: Helicobacter pylori and interleukin-8 in gastric cancer. World J Gastroenterol 19: 8192-8202, 2013.

4. Taylor MA, Parvani JG and Schiemann WP: The pathophysiology of epithelial-mesenchymal transition induced by transforming growth factor-beta in normal and malignant mammary epithelial cells. J Mammary Gland Biol Neoplasia 15: 169-190, 2010.

5. Nitta T, Mitsuhashi T, Hatanaka Y, Miyamoto M, Oba K, Tsuchikawa T, Suzuki Y, Hatanaka KC, Hirano S and Matsuno Y: Prognostic significance of epithelial-mesenchymal transition-related markers in extrahepatic cholangiocarcinoma: Comprehensive immunohistochemical study using a tissue microarray. Br J Cancer 111: 1363-1372, 2014.

6. Thiery JP, Acloque H, Huang RY and Nieto MA: Epithelial-mesenchymal transitions in development and disease. Cell 139: 871-890, 2009.

7. Kim MA, Lee HS, Lee HE, Kim JH, Yang HK and Kim WH: Prognostic importance of epithelial-mesenchymal transition-related protein expression in gastric carcinoma. Histopathology 54: 442-451, 2009.

8. Cao H, Xu W, Qian H, Zhu W, Yan Y, Zhou H, Zhang X, Xu X, Li J, Chen Z and Xu X: Mesenchymal stem cell-like cells derived from human gastric cancer tissues. Cancer Lett 274: 61-71, 2009.

9. Schäffler A and Büchler C: Concise review: Adipose tissue-derived stromal cells - basic and clinical implications for novel cell-based therapies. Stem Cells 25: 818-827, 2007.

10. Sasaki M, Abe R, Fujita Y, Ando S, Inokuma D and Shimizu H: Mesenchymal stem cells are recruited into wounded skin and contribute to wound repair by transdifferentiation into multiple skin cell type. J Immunol 180: 2581-2587, 2008.

11. Spaeth E, Klopp A, Dembinski J, Andreeff M and Marini F: Inflammation and tumor microenvironments: Defining the migratory itinerary of mesenchymal stem cells. Gene Ther 15: 730-738, 2008

12. Quante M, Tu SP, Tomita H, Gonda T, Wang SS, Takashi S, Baik GH, Shibata W, Diprete B, Betz KS, et al: Bone marrow-derived myofibroblasts contribute to the mesenchymal stem cell niche and promote tumor growth. Cancer Cell 19: 257-272, 2011.

13. Glaire MA, El-Omar EM, Wang TC and Worthley DL: The mesenchyme in malignancy: A partner in the initiation, progression and dissemination of cancer. Pharmacol Ther 136: 131-141, 2012.

14. Taichman RS, Wang Z, Shiozawa Y, Jung Y, Song J, Balduino A, Wang J, Patel LR, Havens AM, Kucia M, et al: Prospective identification and skeletal localization of cells capable of multilineage differentiation in vivo. Stem Cells Dev 19: 1557-1570, 2010.

15. Houghton J, Stoicov C, Nomura S, Rogers AB, Carlson J, Li H, Cai X, Fox JG, Goldenring JR and Wang TC: Gastric cancer originating from bone marrow-derived cells. Science 306 : 1568-1571, 2004.

16. Ferrand J, Lehours P, Schmid-Alliana A, Mégraud F and Varon C: Helicobacter pylori infection of gastrointestinal epithelial cells in vitro induces mesenchymal stem cell migration through an NF-кB-dependent pathway. PLoS One 6: e29007, 2011.

17. Qiao C, Xu W, Zhu W, Hu J, Qian H, Yin Q, Jiang R, Yan Y, Mao F, Yang H, et al: Human mesenchymal stem cells isolated from the umbilical cord. Cell Biol Int 32: 8-15, 2008.

18. Zhang Q, Wang M, Huang F, Yang T, Cai J, Zhang X, Zhu W, Qian $\mathrm{H}$ and $\mathrm{Xu}$ W: $H$. pylori infection-induced MSC differentiation into CAFs promotes epithelial-mesenchymal transition in gastric epithelial cells. Int J Mol Med 32: 1465-1473, 2013.

19. Yang T, Zhang X, Wang M, Zhang J, Huang F, Cai J, Zhang Q, Mao F, Zhu W, Qian H and Xu W: Activation of mesenchymal stem cells by macrophages prompts human gastric cancer growth through NF- $\kappa$ B pathway. PLoS One 9: e97569, 2014.

20. Zhou H, Sheng L, Wang H, Xie H, Mu Y, Wang T and Yan J: Anti- $\beta 2$ GPI/ $\beta 2$ GPI stimulates activation of THP- 1 cells through TLR4/MD-2/MyD88 and NF- $\mathrm{BB}$ signaling pathways. Thromb Res 132: 742-749, 2013.

21. Wang M, Cai J, Huang F, Zhu M, Zhang Q, Yang T, Zhang X, Qiang $\mathrm{H}$ and $\mathrm{Xu} \mathrm{W}$ : Pre-treatment of human umbilical cord-derived mesenchymal stem cells with interleukin-6 abolishes their growth-promoting effect on gastric cancer cells. Int J Mol Med 35: 367-375, 2015.

22. Okabe C, Borges RL, de Almeida DC, Fanelli C, Barlette GP, Machado FG, Arias SC, Malheiros DM, Camara NO, Zatz R and Fujihara CK: NF- $\kappa$ B activation mediates crystal translocation and interstitial inflammation in adenine overload nephropathy. Am J Physiol Renal Physiol 305: F155-F163, 2013. 
23. Zhang J, Jiang W and Zuo Z: Pyrrolidine dithiocarbamate attenuates surgery-induced neuroinflammation and cognitive dysfunction possibly via inhibition of nuclear factor $\kappa \mathrm{B}$. Neuroscience 261: 1-10, 2014.

24. Chan J, O'Donoghue K, de la Fuente J, Roberts IA, Kumar S, Morgan JE and Fisk NM: Human fetal mesenchymal stem cells as vehicles for gene delivery. Stem Cells 23: 93-102, 2005.

25. Korkaya H, Liu S and Wicha MS: Breast cancer stem cells, cytokine networks, and the tumor microenvironment. J Clin Invest 121: 3804-3809, 2011

26. Shinagawa K, Kitadai Y, Tanaka M, Sumida T, Kodama M, Higashi Y. Tanaka S, Yasui W and Chayama K: Mesenchymal stem cells enhance growth and metastasis of colon cancer. Int J Cancer 127: 2323-2333, 2010.

27. Yu JM, Jun ES, Bae YC and Jung JS: Mesenchymal stem cells derived from human adipose tissues favor tumor cell growth in vivo. Stem Cells Dev 17: 463-473, 2008.
28. Steeg PS: Tumor metastasis: Mechanistic insights and clinical challenges. Nat Med 12: 895-904, 2006.

29. Christiansen JJ and Rajasekaran AK: Reassessing epithelial to mesenchymal transition as a prerequisite for carcinoma invasion and metastasis. Cancer Res 66: 8319-8326, 2006.

30. Kalluri R and Weinberg RA: The basics of epithelial-mesenchymal transition. J Clin Invest 119: 1420-1428, 2009.

31. Folkman J: Role of angiogenesis in tumor growth and metastasis. Semin Oncol 29: 15-18, 2002.

32. Grant CM and Kyprianou N: Epithelial mesenchymal transition (EMT) in prostate growth and tumor progression. Transl Androl Urol 2: 202-211, 2013.

33. Christiansen JJ and Raiasekaran AK: Reassessing epithelial to mesenchymal transition as prerequisite for carcinoma invasion and metastasis. Cancer Res 66: 8319-8326, 2006.

34. Tye H and Jenkins BJ: Tying the knot between cytokine and toll-like receptor signaling in gastrointestinal tract cancers. Cancer Sci 104: 1139-1145, 2013. 